\title{
Characterization and Testing of Novel Polarized Nanomaterial Textiles for Ultrasensitive Wireless Gas Sensors
}

\author{
Trang Thai*(1) ${ }^{(1)}$ ustin Ratner ${ }^{(1)}$, Wenhua Chen ${ }^{(2)}$, Gerald DeJean ${ }^{(3)}$, and Manos M. Tentzeris ${ }^{(1)}$ \\ (1) GEDC, School of ECE, Georgia Institute of Technology, Atlanta, GA 30332-250, USA \\ (2) State Key Lab on Microwave \& Digital Communication, Tsinghua Univ., Beijing, 100084, P.R.China
}

(3) Microsoft Research, One Microsoft Way, Redmond, WA 98052, USA

Email: trang.thai@gatech.edu

\begin{abstract}
A novel polarized nano-material (PNM) textile is fabricated and characterized at Ka-band $(26.5$ to $40 \mathrm{GHz})$ by rectangular waveguide measurements for three different polarization schemes (crossed, horizontal, and vertical polarized samples). Since carbon nanotubes were found to be ultra sensitive to different gases at extremely low concentration, a very important application is integrated gas sensors that are based on the change in the electrical properties of carbon nanotube materials induced by gas molecule adsorption. However, a systematic design methodology for high frequency gas sensors utilizing carbon nanotube materials is not yet possible due to the lack of indepth knowledge on the material properties before and after being exposed to the gases of interests. In this study, the scattering parameters of PNM textile embedded in waveguides are measured in both room atmosphere and in ammonia/air mixture of 5\% ammonia. The gas measurement show a phase shift of 10 degrees in S11 values. The impedance of the PNMs are computed from the scattering parameters in waveguide measurements, which for the first time experimentally show that CNTs can function as resonators at microwave frequencies.
\end{abstract}

\section{Introduction}

Carbon nanotubes (CNTs) were found to have many excellent characteristics ranging from mechanical, thermal, to electrical properties. Their applications cover numerous interests from gas sensing and shielding to nanotube antennas and other important microwave applications. There have been various measurements and investigations performed on different CNT materials including characterizations and designs for specific applications such as gas sensors [1][2]. However, most of those works take place either at low frequencies or at optical frequencies. Recently there have been some works performed on CNT based materials at higher frequencies up to millimeter wave range [3][4], and these measurements were performed on randomly aligned multiwall carbon nanotubes (MWNTs) based samples. Since the novelty of aligned CNTs have not yet been explored and characterized while their properties promise great applications especially in sensing and microwave circuits, thus in this paper the impedance of a novel CNT material, the Polarized Nano-Material (PNM) textile, is investigated with three different polarization schemes in Ka band (26.5 - 40 $\mathrm{GHz}$ ) to serve various microwave applications and effective material engineering concepts.

Novel Polarized Nano-Material (PNM) textile is formed by drawing many alligned nanotubes from aligned CNT arrays and assembling them into different polarized schemes.
Thus, the final samples become highly polarized and have an excellent shielding effect. Its electric field absorbing properties at $3-7 \mathrm{GHz}$ were presented in [5]. In this work, the electrical properties of this novel PNM textile are pursued at millimeter wave frequencies to reveal the unique behavior of parallel CNTs. While many theoretical studies have predicted the functionality of CNTs as resonators, such a feature has not been experimentally verified at microwave frequency range. The impedance analysis of the PNM material in this paper directly shows for the first time the radiation effect of CNTsexperimentally. In addition, the PNM samples are exposed to ammonia in a high-frequency operation so as their gas sensitivity can be evaluated. As carbon nanotubes were found to be ultra sensitive to different gases such as $\mathrm{NH}_{3}, \mathrm{CO}_{2}, \mathrm{NO}_{\mathrm{X}}$, etc. at part-per-million to part-per-billion levels, they could be a fundamental part of a new disruptive generation of ultrasensitive integrated gas sensors that are based on the change in the electrical properties of carbon nanotube materials induced by gas molecule adsorption. Therefore in this work, the responses of PNM samples in the presence of ammonia gas are also discussed in terms of scattering parameters.

\section{Polarized Nano-material samples and Experiment set up}

The CNTs are grown on a silicon substrate into superaligned nanotube arrays. They are self-assembled into yarns of up to $30 \mathrm{~cm}$ in length. As bundles of CNTs are pulled out from the super aligned CNT arrays, continuous yarns of pure CNTs can be obtained and easily aligned parallel to one another due to van der Waals interactions. The yarns composed of parallel threads that have diameters in the range of several hundreds of nanometers (Fig. 1a) [5]. The width of the yarn depends on the number of threads in the yarn. The CNT yarns were constructed into three schemes of alignment that form three types of polarization as shown in Fig. 1b. There are 20 layers of the PNM textile that were weaved oneby-one on to one waveguide aperture and joined by another identical waveguide section, both are WR-28 operating in Ka-band of 26.5-40 GHz), thus forming a waveguide transmission line as shown in Fig. 2. Scattering parameters of three different polarizations of PNM embedded in the two waveguide sections were measured with the Agilent E8363B PNA. 


\section{Scattering parameters and Impedance Analysis of Polarized Nano-material samples}

\section{A. Scattering parameters and PNM's response to ammonia}

The scattering parameter results are shown in Fig. $3-4$, which are qualitatively similar to those presented previously in [4] on the same PNM textile. Polarization 2 sample has its return loss close to $-20 \mathrm{~dB}$ indicating that little signal is reflected; while the return loss of polarization 1 and 3 is above $-3 \mathrm{~dB}$ showing significant reflection. Due to the polarization schemes, it is expected that most electric field would be blocked by polarization 1 and 3 samples, while polarization 2 sample should show open waveguide characteristic with its $\mathrm{S} 21$ value close to $0 \mathrm{~dB}$. The results presented in Fig. 4 illustrate this principle in excellent agreement. The shielding effect of polarizations 1 and 3 are due to the alignment of CNTs along the electric field polarization, thus the conductivity along the nanotube principle axis cancels out most of the field. Values for Sparameters obtained here in $26.5-40 \mathrm{GHz}$ are slightly different from those presented in [4] at a different range of 3 $-7 \mathrm{GHz}$. This indicates that the PNM materials are highly suitable for wideband applications.

The sample of polarization \#3 was exposed to ammonia (NH3) gas in a glass tube. The gas was premixed with air to make $5 \%$ of concentration. Since the CNTs based materials were reported to have quick response time but slow recovery time to gases [6], the gas exposure performed here lasted for 30 minutes to ensure saturation. The measurements of return loss S11 were performed on the gas exposed sample in the same Ka-band of frequencies. The results before and after ammonia exposure are compared and plotted in Fig. 5 with magnitude of S11 in absolute values and its phase in degrees. There is no observable change in the magnitude of S11, while its phase is shown to be shifted by approximately 10 degrees. This phase shift is similar to the phase shift results obtained by Dragoman et al. reported in Fig. 3 of [3] in the frequency range of $24.5-40 \mathrm{GHz}$. Since the PNM sample was constructed to be in extremely thin film, the change in the dielectric constant (reflected in the S11 magnitude) is more difficult to be observed. It was reported at lower frequencies that the imaginary part of the permittivity (reflected in the phase of S11 values) of the CNTs based materials are altered more significant than the real part of its permittivity (reflected in the magnitude of S11 values) [1], which is in agreement with the results obtained here.

\section{B. Impedance Analysis of PNM thin film}

The impedance of the PNM material can be computed based on S-parameters according to the following equation $[4][7]$.

$$
Z=\sqrt{\frac{(1+S 11)^{2}-(S 21)^{2}}{(1-S 11)^{2}-(S 211)^{2}}}
$$

The real part and imaginary part of the impedance of the PNM layers are shown in Fig. 6. The impedance profiles of polarization 1 and 3 show distinct resonance characteristics of radiator, which can be modeled by an LC lump element circuit. They both resonate at around 31 and $38 \mathrm{GHz}$. These behaviors were predicted in [8] as well as other studies, and although similar behavior was observed in the optical range, this is the first time the collectively resonance behavior of the carbon nanotubes are shown in the microwave range due to highly aligned CNTs in the PNM layers. This would pave the way for further study on the radiation properties of CNTs as they act like resonators. The impedance profile of polarization 2 shows no resonance which confirms again the prediction that most currents are induced along the nanotube main axis instead of the axial axis that is circular around the main axis.

\section{Conclusions}

Novel PNM textile thin film samples were fabricated, constructed, and characterized at millimeter wave frequencies $(26.5$ - $40 \mathrm{GHz})$, and their impedance properties were presented. The results show that the PNM is an excellent absorber for wideband applications; the highly polarized materials can be utilized for polarization detection and sensing applications. The measurements of PNM polarization \#3 in exposure to ammonia of 5\% concentration show a shift of 10 degrees in phase, while the magnitude change is observable. Future work will include further investigation on the gas sensitivity of PNM textile at millimeter-wave frequencies to further enhance and establish a solid foundation for prospective high-frequency ultrasensitive wireless gas sensors. The impedance results of the PNM textile verify that CNTs can function as resonators at microwave frequencies, hence, paving ways for further theoretical and experimental studies on these nanomaterials.

\section{Acknowledgments}

The authors are grateful to the help of Prof. Jiri Janata and $\mathrm{PhD}$ candidate Alex Jonke of School of Chemistry and Biochemistry, Georgia Tech in the gas experiments. The authors would also like to thank Dr. John Cressler and PhD candidate Tushar Thrivikraman for the help in measurements. Finally, the authors would like to acknowledge the support of Georgia Electronic Design Center.

\section{References}

1. K. G. Ong, K. Zeng, C. A. Grimes, "A wireless, passive carbon nanotube-based gas sensor," IEEE Sens. J., 2, pp 82-88 (2002).

2. S. Chopra, A.Pham, J. Gaillard, A. Parker, A. M. Rao, "Carbon-nanotube-based resonant-circuit sensor for ammonia,” Appl. Phys. Lett. 80, 4632 (2002).

3. M. Dragoman et.al., "Millimeter wave carbon nanotube gas sensor," App. Phy. Lett. 101, 106103 (2007).

4. L. Wang, R. Zhou, H. Xin, "Microwave (8-50 GHz) Characterization of Multiwalled Carbon Nanotube Papers Using Rectangular Waveguides," IEEE Trans. Micr. Theory Tech., Vol. 56, No. 2, February 2008, Pages(s): 499-506.

5. W. Chen, Z. Zhang, Z. Feng, Y. Chen, K. Jiang, S. Fan, M. Iskander, "Measurement of Polarized Nano-Material (PNM) for Microwave Applications," IEEE MTT-S Int. Micr. Symp. Dig. 15-20 June 2008, Page(s): 336-339. 
6. N. Sinha, J. Ma, J. Yeow, "Carbon Nanoube-Based Sensors," J. Nanosci. Nanotechnol. 2006, Vol. 6, No. 3, pp 573-590 (2006).

7. X. Chen, T. Grzegorezyk, B. Wu, J. Pacheco, J. Kong, "Robust method to retrieve the constitutive effective parameters of metamaterials," Phys. Rev. E 70, 016608 (2004).
8. G. W. Hanson, "Fundamental Transmitting Properties of Carbon Nanotube Antennas," IEEE Trans. Ant. And Prop., Vol. 53, No. 11, November 2005, Page(s): 34263435.

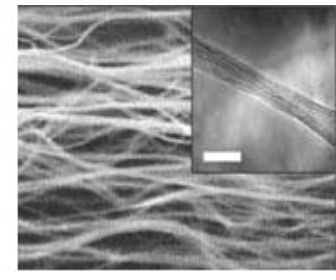

(a)

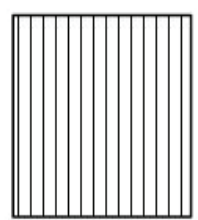

Polarization \#1 Polarization \#2 Polarization \#3

(b)

Fig. 1. (a) Capture of PNM textile, and (b) Different polarization schemes. [4]

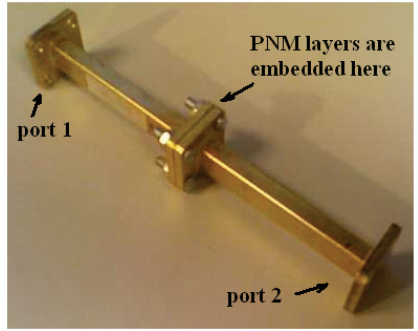

Fig. 2. PNM is embedded in waveguide sections for measurements of S-parameter.
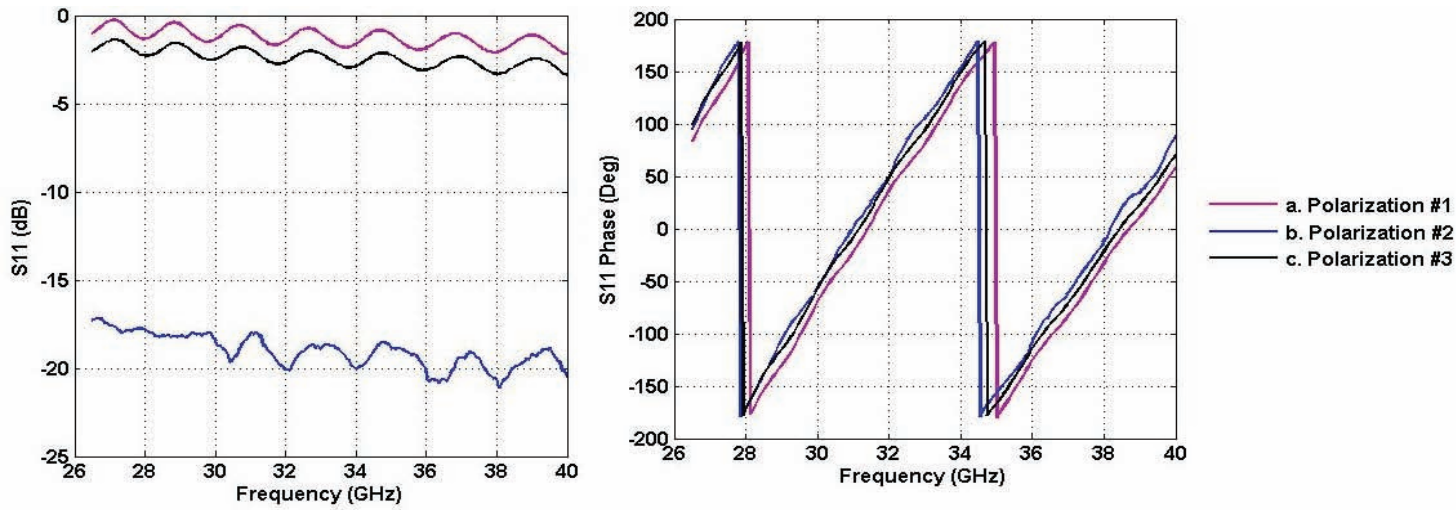

Fig. 3. S11 magnitude and phase of the embedded PNM layers.
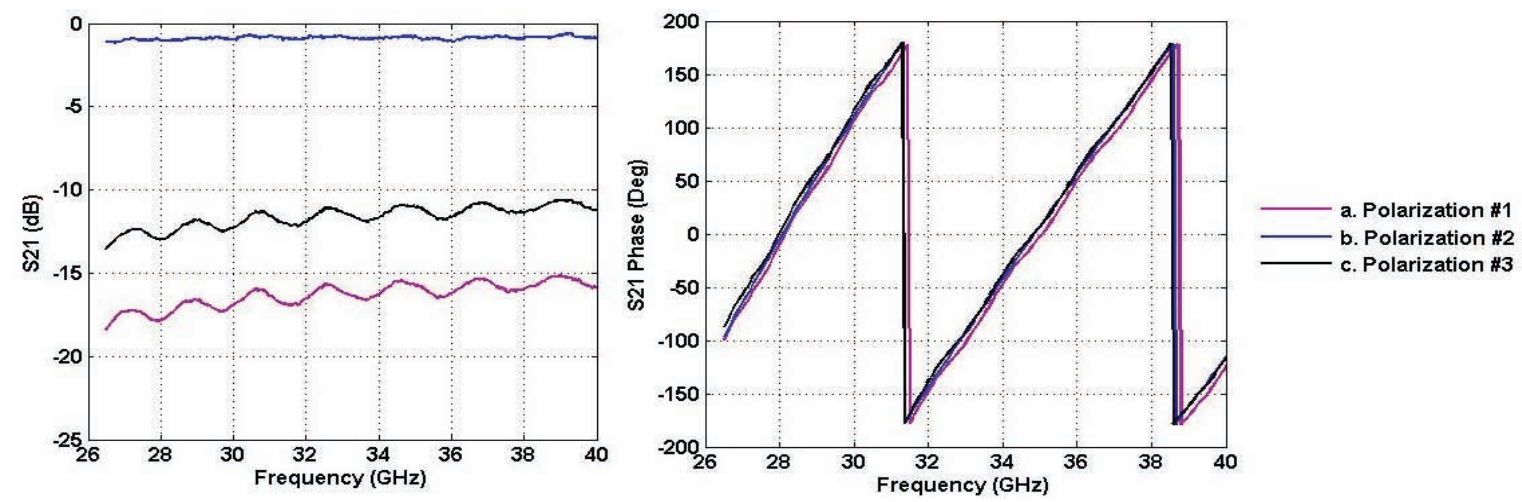

Fig. 4. S21 magnitude and phase of the embedded PNM layers. 

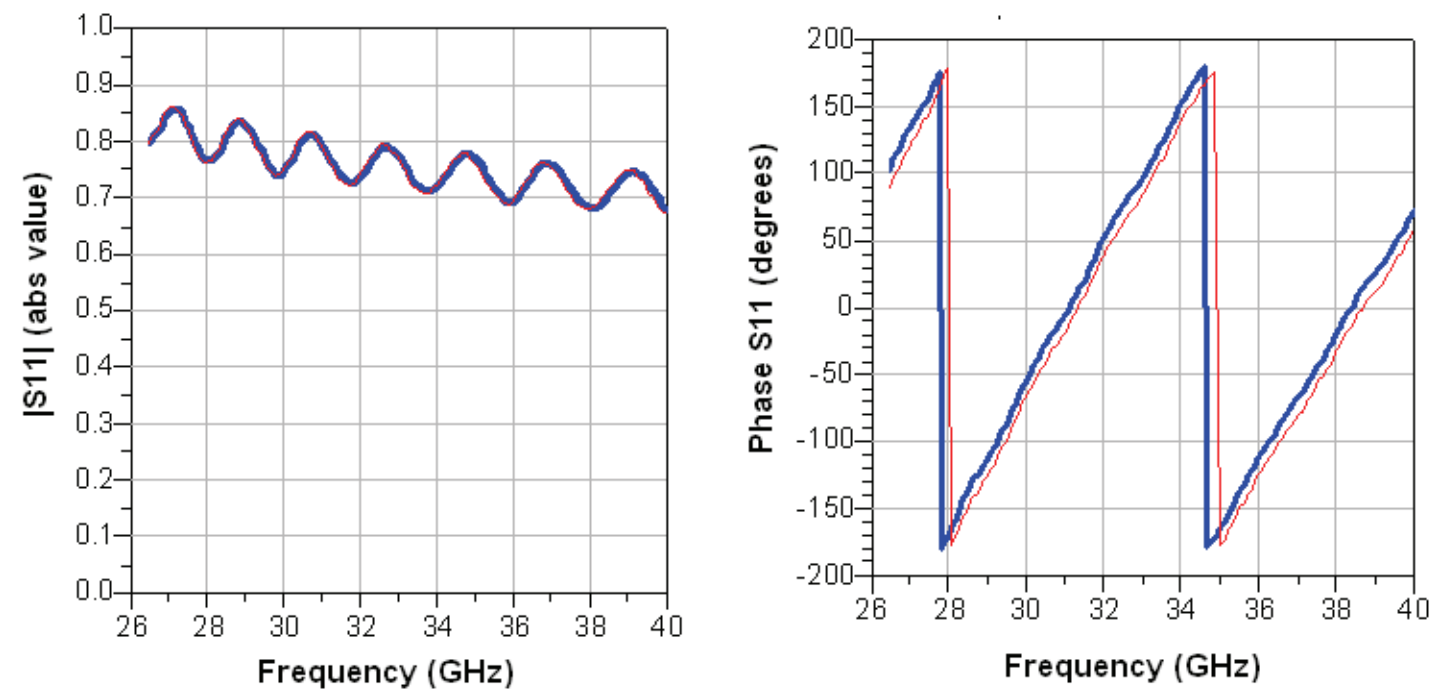

Fig. 5. Magnitude (absolute value) and Phase (degrees) of S11 of PNM polarization \#3 sample before (red line) and after (blue line) exposure to Ammonia of 5\%.
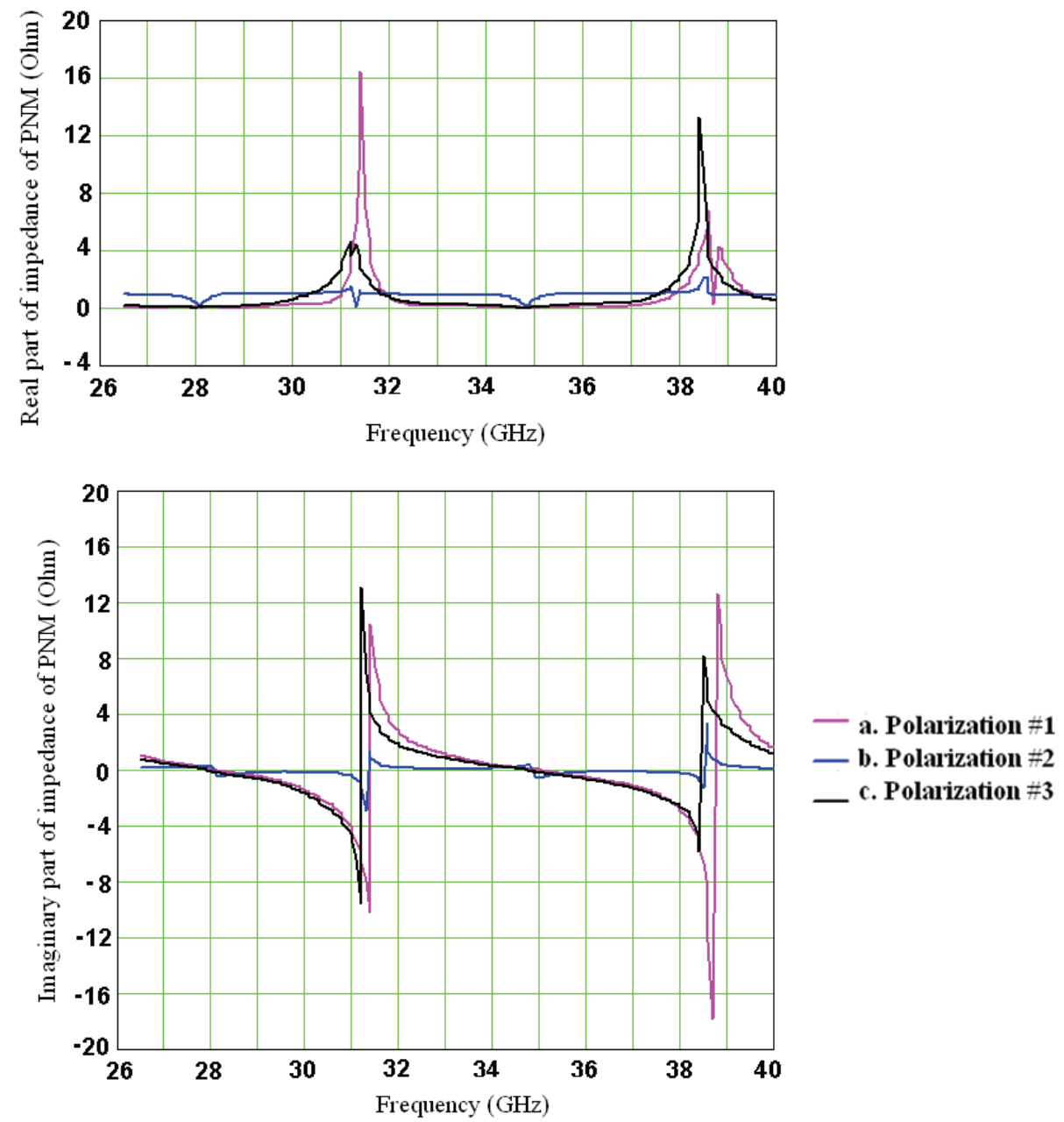

Fig. 6. Real and Imaginary part of the impedance of the PNM material. 